\title{
Isavuconazole and Liposomal Amphotericin B as Successful Combination Therapy of Refractory Invasive Candidiasis in a Liver Transplant Recipient: A Case Report and Literature Review
}

\author{
Georgios Odysseos • Ulrich Mayr • Gabor Bozsaki • Christian Seidensticker • \\ Ursula Ehmer • Roland M. Schmid • Tobias Lahmer • Veronika Dill (i)
}

Received: 11 June 2021/Accepted: 15 October 2021 / Published online: 31 October 2021

(C) The Author(s) 2021

\begin{abstract}
Invasive fungal infections in liver transplant recipients are associated with elevated morbidity and mortality and pose a challenge to the treating physicians. Despite of lacking clinical data, the use of antifungal combination therapy is often considered to improve response rates in an immunocompromised patient population. We herein report a case of refractory invasive candidiasis in a liver transplant recipient treated successfully with a combination of isavuconazole und high-dose liposomal amphotericin B. The antimycotic combination treatment was able to clear a bloodstream infection with $C$. glabrata and led to regression of bilomas among tolerable side effects. The use of the above-mentioned antifungal combination therapy in a liver transplant recipient has not been reported previously. This case highlights the efficacy and safety of antifungal combination therapy in immunocompromised patients with refractory invasive candidiasis.
\end{abstract}

G. Odysseos · U. Mayr - G. Bozsaki - C. Seidensticker ·

U. Ehmer · R. M. Schmid · T. Lahmer

Klinik und Poliklinik für Innere Medizin II, Klinikum rechts der Isar der Technischen Universität München, Munich, Germany

V. Dill ( $₫)$

Klinik und Poliklinik für Innere Medizin III, Klinikum rechts der Isar der Technischen Universität München, Ismaninger Straße 22, 81675 Munich, Germany

e-mail: veronika.dill@tum.de
Keywords Candidemia - Invasive candidiasis . Liver transplantation - Antifungal combination therapy · Isavuconazole $\cdot$ Liposomal amphotericin B

\section{Introduction}

Liver transplantation (LT) is a well-established clinical procedure for the treatment of terminal liver diseases. Survival rates after LT have improved significantly over the past decades, reaching $85 \%$ at 1 year and $73 \%$ at 5 years considering the last 10 -year period [1]. This accomplishment is mainly achieved by the introduction of new immunosuppressive agents, the improvement of surgical techniques and the early diagnosis and treatment of complications [2]. Irrespective of the administration of an antifungal prophylaxis, invasive fungal infections (IFI) can be observed in approximately 5-7\% of liver transplant recipients $[3,4]$. Previous studies reported that IFI in liver transplant recipients occurred mainly in the early phase after LT [5] and frequently in patients with renal insufficiency, vascular/biliary complications and retransplantation [6]. Invasive candidiasis represents the most common IFI subtype and refers to candidemia, a bloodstream infection with Candida species (spp.) as well as deep-seated infection, with or without candidemia [7]. 
After diagnosis of candidemia antifungal therapy should be initiated immediately with an appropriate antifungal agent from the substance groups of azoles, echinocandins and liposomal amphotericin B. Despite proper therapy, candidemia causes significantly elevated morbidity and mortality in this immunocompromised patient population [6]. Consequently, IFI remain a challenging situation for the treating physicians and the use of antifungal combination therapy is often considered to improve response rates. Nevertheless, there is only limited clinical data available to support antifungal combination therapy over singleagent treatment in patients with invasive candidiasis [8]. Moreover, the usage of antimycotic combination treatment has never been evaluated as first-line or salvage therapy in liver transplant recipients [9]. To the best of our knowledge, this is the first report describing the successful combination of isavuconazole (ISA) and liposomal amphotericin B (AMB) in a liver transplant recipient with refractory invasive candidiasis.

\section{Case Report}

A 52-year old female, who underwent LT past 3 months due to primary biliary cirrhosis, was admitted because of fever up to $40{ }^{\circ} \mathrm{C}$. In the past history, short term after LT, increased cholestasis parameters and transaminases had been noted. Endoscopic retrograde cholangiography (ERC) had demonstrated ischemic-type biliary lesions, characterized by intrahepatic bile duct strictures, in the absence of perfusion restrictions. At that time a biliary cannulation had been performed. Post intervention both liver and cholestasis parameters had decreased. An acute rejection of the transplanted liver had been ruled out by biopsy. The post-transplant immunosuppression consisted of mycophenolate mofetil (MMF) and tacrolimus. Due to the absence of high-risk factors for fungal infections $[5,6]$, antifungal prophylaxis had not been administered post-transplant.

The physical examination on the day of admission to our hospital was unremarkable. The patient was hemodynamically stable, had normal breathing sounds, no cardiac murmur and the abdomen reconciled normal. Blood results showed high inflammation markers with elevated CRP, procalcitonin and IL- 6 as well as marginally elevated transaminases with normal cholestasis parameters. An abdominal ultrasound examination showed slightly dilated peripheral bile ducts in the absence of focal lesion. Therapeutic ERC with double pigtail stent replacement was performed. Two of three blood cultures from admission day detected colonies of Enterococcus faecalis and Enterococcus casseliflavus. With similar detection of both bacteria in the bile culture, a biliary focus seemed very likely. Broad spectrum antibiotics with meropenem and linezolid were given. During hospitalization the patient developed infection-associated progressive neutropenia (neutrophil nadir $0.74 \mathrm{G} / 1$ on day 4). As a consequence, MMF was paused and immunosuppression was adapted with tacrolimus and prednisolone (short-term administration of a maximum of $20 \mathrm{mg}$ prednisolone per day and gradual reduction to a maintenance dose of $5 \mathrm{mg}$ per day). Of note, leukocytes increased within a week after drug discontinuation.

Systemic candidiasis was diagnosed for the first time in the follow-up blood cultures on hospital day 8 based on the evidence of $C$. glabrata (Table 1A). Candida sepsis was diagnosed due to organ dysfunction with a Sequential Organ Failure Assessment (SOFA) score $>2$ points. Shortly after the diagnosis, an intravenous antifungal therapy with caspofungin (CAS; $70 \mathrm{mg}$ loading dose on day 1 , followed by $50 \mathrm{mg}$ daily thereafter) was initiated. A biloma in liver segment VI/VII was detected for the first time on day 10 by abdominal sonography. Laboratory findings consisted of slightly increasing transaminases (Fig. 1). An endophthalmitis was ruled out within a week after diagnosis. Extensive imaging, including echocardiography, orthopantomography and magnetic resonance imaging (MRI) of the spine did not reveal any evidence of an infection focus with $C$. glabrata. The peripheral venous catheter was changed regularly. Nevertheless, blood cultures, repeatedly taken every 24-48 h, remained positive for C. glabrata.

Due to persistent fungemia and recurrent episodes of fever, the intravenous antimycotic therapy was converted to isavuconazole (ISA; $200 \mathrm{mg}$ three-times daily for two days, followed by $200 \mathrm{mg}$ once daily) on hospital day 18. This substance was chosen contrary to other triazoles due to increased creatinine levels and hepatic enzymes during candidemia. Shortly after switching to isavuconazole, both aspartate aminotransferase (AST) and alanine aminotransferase (ALT) decreased (Fig. 1). However, further 
Table 1 Antimycotic sensitivity testing for C. glabrata categorized as susceptible (S) or susceptible at increased exposure (IE) towards the indicated antifungal agents (A) on hospital day 8 and (B) on hospital day 26. Minimum inhibitory concentration (MIC) breakpoints (mg/L) were determined according to the recommendations of the European Committee on Antimicrobial Susceptibility Testing (EUCAST)

\begin{tabular}{lll}
\hline Antifungal agent & (A) Sensitivity testing on day 8 & (B) Sensitivity testing on day 26 \\
\hline Amphotericin B & $\mathrm{S}(\leq 0,25 \mathrm{mg} / \mathrm{L})$ & $\mathrm{S}(1 \mathrm{mg} / \mathrm{L})$ \\
Voriconazol & $\mathrm{IE}(0,25 \mathrm{mg} / \mathrm{L})$ & $\mathrm{IE}(0,25 \mathrm{mg} / \mathrm{L})$ \\
Caspofungin & $\mathrm{S}(\leq 0,12 \mathrm{mg} / \mathrm{L})$ & $\mathrm{S}(0,5 \mathrm{mg} / \mathrm{L})$ \\
Flucytosin & $\mathrm{IE}(\leq 1 \mathrm{mg} / \mathrm{L})$ & $\mathrm{IE}(\leq 1 \mathrm{mg} / \mathrm{L})$ \\
Micafungin & $\mathrm{S}(\leq 0,06 \mathrm{mg} / \mathrm{L})$ & $\mathrm{S}(\leq 0,06 \mathrm{mg} / \mathrm{L})$
\end{tabular}
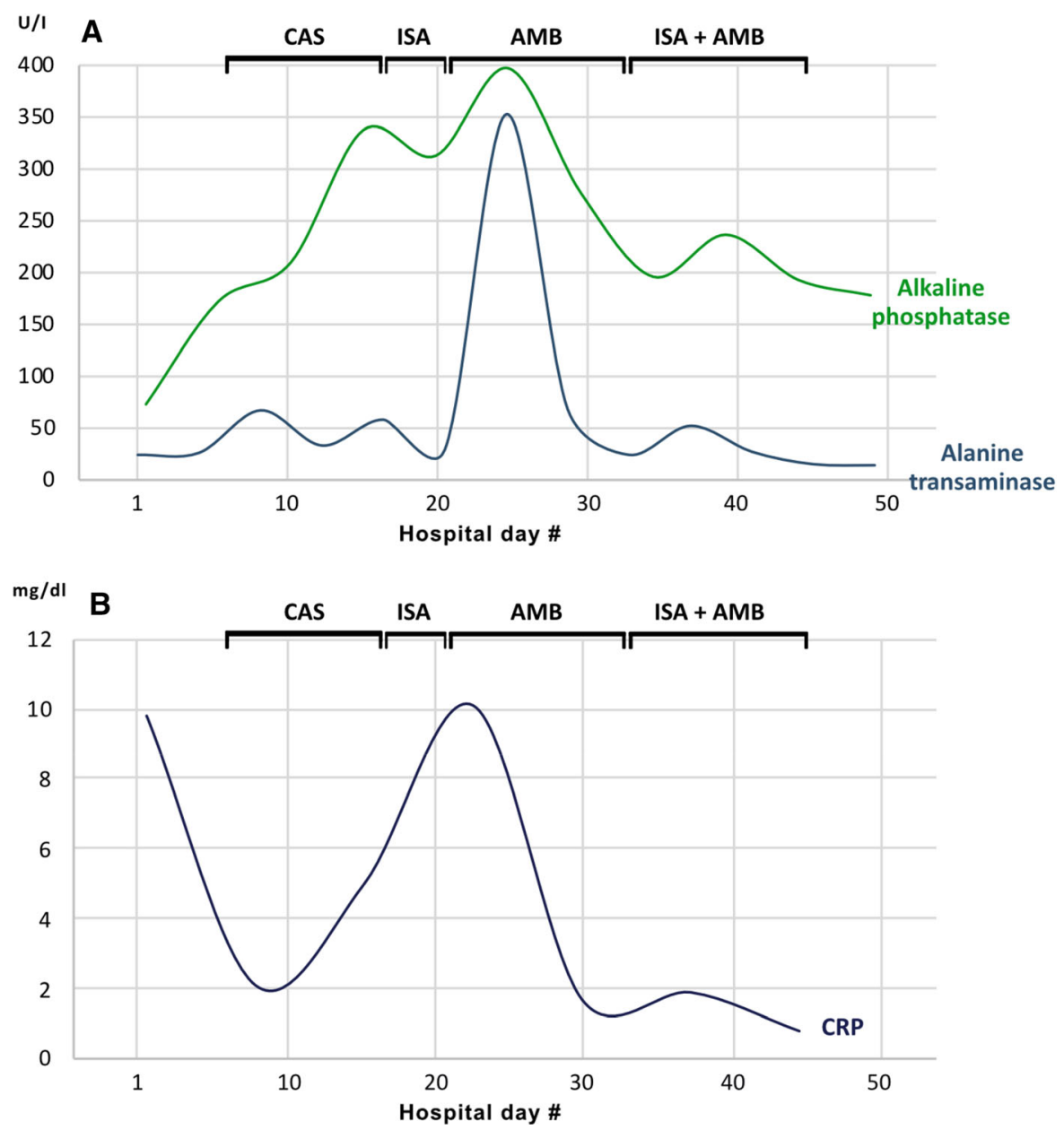

Fig. 1 Longitudinal changes of alkaline phosphatase, alanine transaminase (A) and the inflammation marker C-reactive protein (CRP) (B) during the patient's hospitalization. The antifungal agents used in the certain period are marked

MRI imaging on hospital day 19 showed new bilomas with a maximum size of $23,8 \times 15,2 \mathrm{~mm}$ (Fig. 2) in accordingly (CAS: caspofungin, ISA: isavuconazole, AMB: liposomal amphotericin B). Normal ranges for laboratory values: Alkaline phosphatase 40-129 U/I, alanine transaminase 10-50 U/I, CRP $<0,5 \mathrm{mg} / \mathrm{dl}$

liver segments VII und VIII and an increasing expansion of bile ducts. Cholestasis was thoroughly 

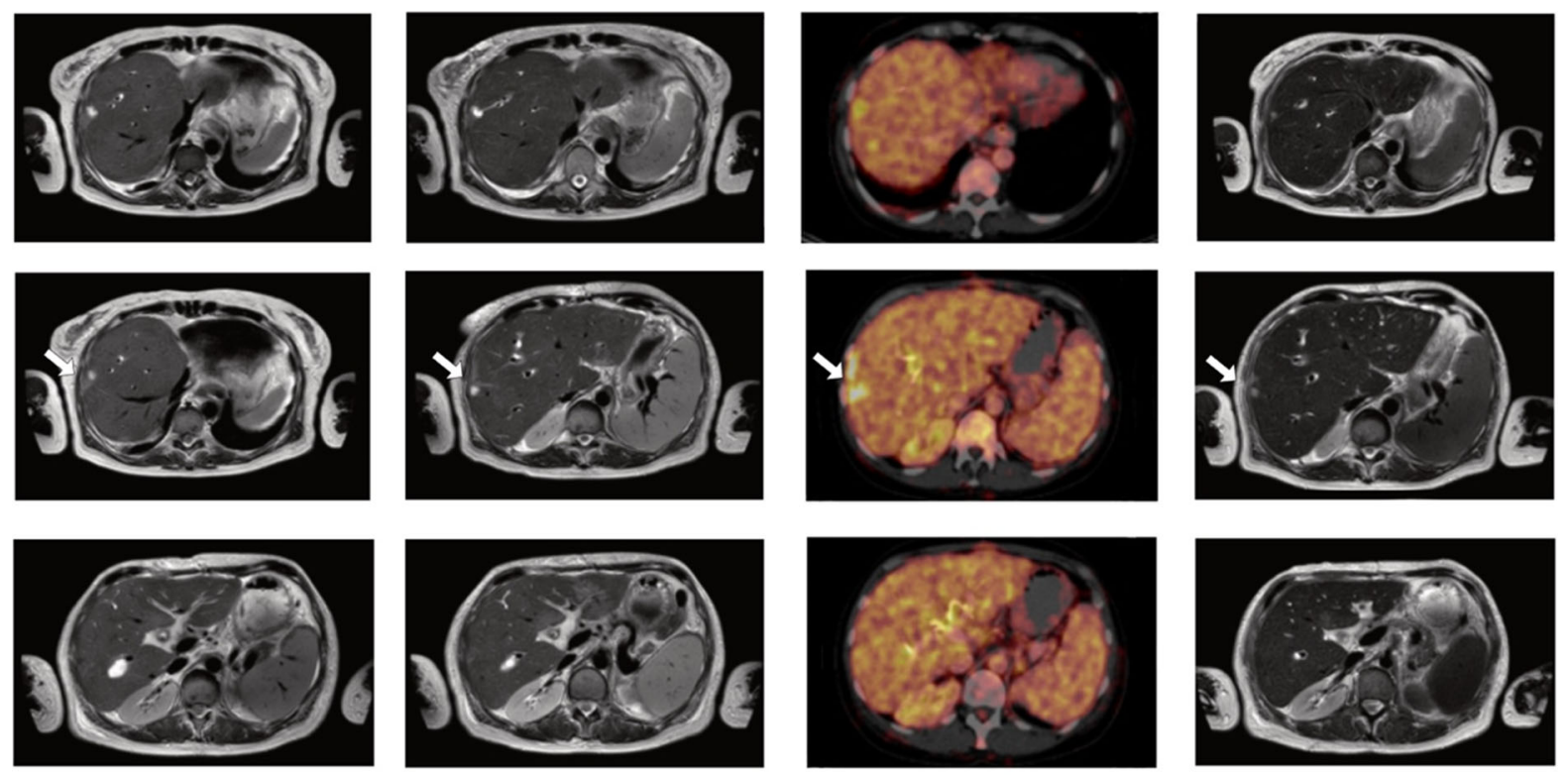

Hospital Day 19

Hospital Day 27

Hospital Day 32

Hospital Day 53

Fig. 2 Image-based morphological monitoring of bilomas during the patient's hospitalization at day 19 (MRI), day 27 (MRI), day 32 (PET/CT) and day $53(\mathrm{MRI})$

reassessed through another ERC on day 21 demonstrating anastomotic stricture and intrahepatic stenosis. Because of complicated structural conditions in the transplanted liver, stent replacement was not possible. Bile drainage was optimized by implantation of three new stents. A percutaneous transhepatic biliary drainage was not required, since internal biliary drainage was possible through an ERC [10].

With persisting positive blood cultures and high systemic inflammation markers (Fig. 1), the antifungal therapy was changed to liposomal amphotericin B (AMB) on hospital day 22 at a daily dose of $3 \mathrm{mg} / \mathrm{kg}$ body weight. Nevertheless, candidemia perseverated despite test-regulated therapy (Table 1B). A follow-up MRI scan of the liver revealed size-progressive dimensioning of bilomas in liver segment VIII with also increasing changes in local liver parenchyma. Increased metabolic activity of the known lesions in liver segment VIII were demonstrated by positron emission tomography-computed tomography (PET/ CT), consistent with ascending cholangitis (Fig. 2). There was no evidence of any further metabolically active sites. Due to the complex anatomical conditions of the transplanted liver, further intervention by ERC was decided to be forgone.
Subsequently we initiated an intensified dual antifungal therapy with high doses of liposomal amphotericin B (daily dose of $6 \mathrm{mg} / \mathrm{kg}$ of body weight) and isavuconazole (200 mg three-times daily for two days, followed by $200 \mathrm{mg}$ once daily) on hospital day 34. From the following day on, all taken blood cultures remained sterile. Interestingly, liver enzymes decreased after starting dual therapy and despite the increased doses of liposomal amphotericin B in the combination regimen compared to monotherapy (Fig. 1). The intravenous antifungal combination therapy was continued for 14 days after the first negative blood culture and stopped afterwards. Six days after completion of therapy, a follow-up MRI scan of the liver was performed, where cholangitis and bilomas showed to be regressed (Fig. 2). It should be noted that during the course of the infection, creatinine levels increased, but remained stable with absence of oliguria or hyperkalemia during the further course of hospitalization.

The patient was hemodynamically stable during the entire stay at our normal ward and discharged without any further complication. Ten days after discontinuation of dual antifungal therapy an antifungal prophylaxis was administered with posaconazole $(300 \mathrm{mg}$ twice on day 1 , followed by $300 \mathrm{mg}$ once a day for 
five weeks), since this substance has been shown to provide high quality evidence in preventing IFI among immunocompromised patients [11]. Follow-up imaging was performed six weeks after discharge and showed further regression of bilomas compared to the previous examination (Fig. 3). A PET/CT demonstrated lacking metabolic activity of the formerly hypermetabolic sites, consistent with a reduced inflammatory activity. Furthermore, there was no evidence of $C$. glabrata in the follow-up blood cultures. Of note, creatinine levels had decreased subsequently and were only slightly elevated at the follow-up visit.

\section{Discussion}

The reported case represents a typical example for IFI in immunocompromised patients. IFI occur in approximately 5-7\% of liver transplant recipients [4] and are predominantly caused by infections with Candida spp. during early post-LT period [6]. With a mortality rate of $30.9 \%$ invasive candidiasis is a lifethreatening complication in liver transplant recipients [12] and a demanding task for clinicians.

After diagnosis of candidemia it is essential to immediately initiate antifungal therapy. Treatment with an echinocandin (e.g., caspofungin: loading dose $70 \mathrm{mg}$, then $50 \mathrm{mg}$ daily) is considered first-line therapy. These substances offer a favorable safety profile, good tolerability and early fungicidal activity, especially in regard to the recent development of azole-resistant Candida spp. [8]. Fluconazole can be used instead of an echinocandin in selected patients, including non-neutropenic patients who are not critically ill [8]. Supplementary, in patients with decompensated liver disease or high-risk liver transplant with proven or probable IFI, treatment with anidulafungin
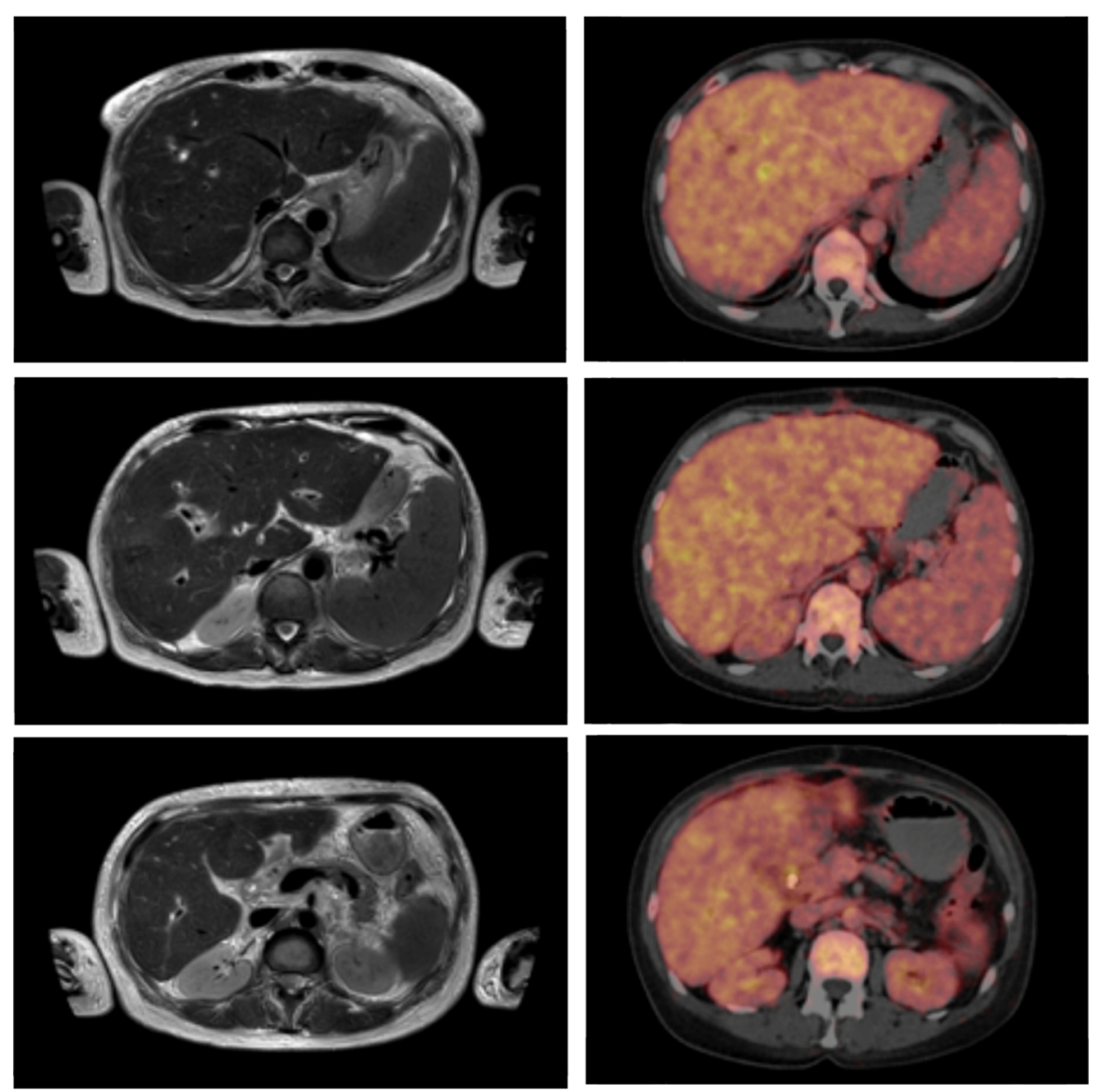

Fig. 3 Follow-up imaging by MRI and PET/CT six weeks after discharge 
demonstrated efficacy and well-tolerated side effects [13]. In patients with azole- and echinocandin-resistant Candida infections, liposomal amphotericin B (3-5 mg/kg daily) can be used alternatively [8]. Of note, this antifungal agent caused more infusionrelated events and nephrotoxicity than caspofungin [14]. In our patient isavuconazole, a new extended-spectrum triazole, was used because of its favorable tolerability profile, less concerns for nephrotoxicity and similar activity against most Candida spp. when compared to other azoles [8, 15-17]. The ACTIVE trial did not show non-inferiority of isavuconazole to caspofungin for first-line treatment of invasive candidiasis [18]. Isavuconazole is approved by the European Medicines Agency (EMA) and the U.S. Food and Drug Administration (FDA) for the treatment of invasive aspergillosis and invasive mucormycosis [17, 19-21]. In general, antimycotic therapy should be continued with a minimum duration of two weeks after documented clearance in the bloodstream [8]. Besides, an adjustment of immunosuppression is recommended [22].

The elevated mortality rate of IFI in immunocompromised patients has led to significant clinical interest in antifungal combination therapy [9]. Due to synergistic interaction between antifungal drugs, antimycotic combination therapy offers a wide spectrum of antifungal activity. Moreover, lower doses of application might reduce the development of resistance. Although previous studies have shown a tolerable side effect profile of dual antifungal therapy [23], such therapy remains controversial [23, 24]. For the purpose of this case, we are going to focus on publications and evidence for the use of antifungal combination therapy in candidemia and invasive candidiasis. A randomized and blinded multicenter trial from Rex et al. showed that the combination of fluconazole and liposomal amphotericin B was not antagonistic compared to fluconazole alone, and the combination trended toward improved success and faster clearance from the bloodstream infection with Candida spp. [25]. An older randomized study from Abele-Horn et al. demonstrated that the treatment of systemic Candida infections with amphotericin B and 5-flucytosine in intensive care patients was more effective than fluconazole alone [26]. It should be mentioned that in this study monotherapy with fluconazole was associated with less toxicity than combined therapy. In one report, fluconazole-resistant oropharyngeal candidiasis was successfully treated with a combination of fluconazole and terbinafine [27].

On the contrary, in vitro combination of isavuconazole and amphotericin B was consistently antagonistic against $C$. glabrata [28]. The triazole-polyene antagonism may be understood by modification of the ergosterol target by the azoles, decreasing amphotericin B activity [24]. Nevertheless, a multilaboratory study from Chaturvedi et al. showed a synergistic effect of posaconazole and amphotericin B against $C$. glabrata [29]. Moreover, in vitro studies demonstrated synergistic activity for the combination of isavuconazol and micafungin against $C$. albicans [28] and for the combination of micafungin with fluconazole and voriconazole against $C$. glabrata [30] supporting synergism of triazoles and echinocandins against Candida spp. [31]. The various results of in vitro cotreatment can be attributed to different experimental settings. Nonetheless, the correlation between laboratory studies and clinical outcome of antifungal combination therapy is not yet determined and randomized clinical studies showed that in vitro antagonism does not necessarily correlate in the clinical setting [25].

Until now, the clinical efficiency of a combination of azoles, specifically isavuconazole, and liposomal amphotericin B has not yet been sufficiently studied. In particular, clinical studies on immunocompromised patients are lacking. Some case reports have already shown a successful combination of isavuconazole and liposomal amphotericin B in disseminated mucormycosis $[32,33]$. To the best of our knowledge, this is the first report illustrating successful dual antimycotic therapy with isavuconazole and liposomal amphotericin B for refractory invasive candidiasis in a liver transplant recipient. In the reported patient, this combination therapy led to clearance of Candida bloodstream infection and complete remission of bilomas in MRI and PET/CT imaging. Of note, this therapy was safely applicable in a patient with hepatic and renal impairment among tolerable side effects.

\section{Conclusion}

Invasive fungal infections (IFI) in immunocompromised patients represent a very challenging situation for physicians. This report clarifies that antifungal cotreatment with isavuconazole (ISA) and liposomal 
amphotericin B (AMB) offers efficacy and a favorable side effect profile in a liver transplant recipient with invasive candidiasis. Antimycotic combination therapy might thus be a promising approach for persistent candidemia in immunocompromised patients. However, further investigation is needed to confirm the positive impact of dual antifungal treatment in this patient population.

Funding Open Access funding enabled and organized by Projekt DEAL.

\section{Declarations}

Conflict of interest The authors declare that they have no conflict of interest.

Ethical Approval All procedures were performed as per the institutional guidelines and in concordance with the Declaration of Helsinki. Written informed consent was obtained from our patient.

Open Access This article is licensed under a Creative Commons Attribution 4.0 International License, which permits use, sharing, adaptation, distribution and reproduction in any medium or format, as long as you give appropriate credit to the original author(s) and the source, provide a link to the Creative Commons licence, and indicate if changes were made. The images or other third party material in this article are included in the article's Creative Commons licence, unless indicated otherwise in a credit line to the material. If material is not included in the article's Creative Commons licence and your intended use is not permitted by statutory regulation or exceeds the permitted use, you will need to obtain permission directly from the copyright holder. To view a copy of this licence, visit http://creativecommons.org/licenses/by/4.0/.

\section{References}

1. Adam R, et al. Evolution of indications and results of liver transplantation in Europe. A report from the European Liver Transplant Registry (ELTR). J Hepatol. 2012;57(3):675-88.

2. Dutkowski $P$, et al. Current and future trends in liver transplantation in Europe. Gastroenterology. 2010;138(3):802-9.

3. Eschenauer GA, et al. Targeted versus universal antifungal prophylaxis among liver transplant recipients. Am J Transplant. 2015;15(1):180-9.

4. Winston DJ, et al. Randomized, double-blind trial of anidulafungin versus fluconazole for prophylaxis of invasive fungal infections in high-risk liver transplant recipients. Am J Transplant. 2014;14(12):2758-64.

5. Ferrarese A, et al. Invasive fungal infection before and after liver transplantation. World J Gastroenterol. 2020;26(47):7485-96.
6. Rabkin JM, et al. Association of fungal infection and increased mortality in liver transplant recipients. Am J Surg. 2000;179(5):426-30.

7. Pappas PG, et al. Invasive candidiasis. Nat Rev Dis Primers. 2018;4:18026.

8. Pappas PG, et al. Clinical practice guideline for the management of candidiasis: 2016 update by the infectious diseases society of America. Clin Infect Dis. 2016;62(4):e1-50.

9. Singh N, et al. Antifungal management practices in liver transplant recipients. Am J Transplant. 2008;8(2):426-31.

10. Thuluvath PJ, Atassi T, Lee J. An endoscopic approach to biliary complications following orthotopic liver transplantation. Liver Int. 2003;23(3):156-62.

11. Wong TY, et al. Efficacy and safety of posaconazole for the prevention of invasive fungal infections in immunocompromised patients: a systematic review with meta-analysis and trial sequential analysis. Sci Rep. 2020;10(1):14575.

12. Andes DR, et al. The epidemiology and outcomes of invasive Candida infections among organ transplant recipients in the United States: results of the Transplant-Associated Infection Surveillance Network (TRANSNET). Transpl Infect Dis. 2016;18(6):921-31.

13. Verma A, et al. Safety and efficacy of anidulafungin for fungal infection in patients with liver dysfunction or multiorgan failure. Open Forum Infect Dis. 2017;4(1):ofw241.

14. Moen MD, Lyseng-Williamson KA, Scott LJ. Liposomal amphotericin B: a review of its use as empirical therapy in febrile neutropenia and in the treatment of invasive fungal infections. Drugs. 2009;69(3):361-92.

15. Falci DR, Pasqualotto AC. Profile of isavuconazole and its potential in the treatment of severe invasive fungal infections. Infect Drug Resist. 2013;6:163-74.

16. Ordaya EE, Alangaden GJ. Real-Life Use of Isavuconazole in Patients Intolerant to Other Azoles. Clin Infect Dis. 2016;63(11):1529-30.

17. Maertens JA, et al. Isavuconazole versus voriconazole for primary treatment of invasive mould disease caused by Aspergillus and other filamentous fungi (SECURE): a phase 3 , randomised-controlled, non-inferiority trial. Lancet. 2016;387(10020):760-9.

18. Kullberg BJ, et al. Isavuconazole versus caspofungin in the treatment of candidemia and other invasive candida infections: the ACTIVE Trial. Clin Infect Dis. 2019;68(12):1981-9.

19. Miceli MH, Kauffman CA. Isavuconazole: a new broadspectrum triazole antifungal agent. Clin Infect Dis. 2015;61(10):1558-65.

20. Marty FM, et al. Isavuconazole treatment for mucormycosis: a single-arm open-label trial and case-control analysis. Lancet Infect Dis. 2016;16(7):828-37.

21. Ananda-Rajah MR, Kontoyiannis D. Isavuconazole: a new extended spectrum triazole for invasive mold diseases. Future Microbiol. 2015;10(5):693-708.

22. Shepshelovich D, et al. Immunosuppression reduction in liver and kidney transplant recipients with suspected bacterial infection: a multinational survey. Transpl Infect Dis. 2019;21(5):13134.

23. Singh N, Husain S. Invasive aspergillosis in solid organ transplant recipients. Am J Transplant. 2009;9(Suppl 4):S180-191. 
24. Baddley JW, Pappas PG. Antifungal combination therapy: clinical potential. Drugs. 2005;65(11):1461-80.

25. Rex JH, et al. A randomized and blinded multicenter trial of high-dose fluconazole plus placebo versus fluconazole plus amphotericin B as therapy for candidemia and its consequences in nonneutropenic subjects. Clin Infect Dis. 2003;36(10):1221-8.

26. Abele-Horn $\mathrm{M}$, et al. A randomized study comparing fluconazole with amphotericin B/5-flucytosine for the treatment of systemic Candida infections in intensive care patients. Infection. 1996;24(6):426-32.

27. Ghannoum MA, Elewski B. Successful treatment of fluconazole-resistant oropharyngeal candidiasis by a combination of fluconazole and terbinafine. Clin Diagn Lab Immunol. 1999;6(6):921-3.

28. Katragkou A, et al. In vitro combination therapy with isavuconazole against Candida spp. Med Mycol. 2017;55(8):859-68.

29. Chaturvedi V, et al. Multilaboratory testing of two-drug combinations of antifungals against Candida albicans, Candida glabrata, and Candida parapsilosis. Antimicrob Agents Chemother. 2011;55(4):1543-8.
30. Nishi I, et al. In vitro antifungal combination effects of micafungin with fluconazole, voriconazole, amphotericin B, and flucytosine against clinical isolates of Candida species. J Infect Chemother. 2009;15(1):1-5.

31. Roling EE, et al. Antifungal activities of fluconazole, caspofungin (MK0991), and anidulafungin (LY 303366) alone and in combination against Candida spp. and Crytococcus neoformans via time-kill methods. Diagn Microbiol Infect Dis. 2002;43(1):13-7.

32. Cornu M, et al. Successful outcome of disseminated mucormycosis in a 3-year-old child suffering from acute leukaemia: the role of isavuconazole? A case report. BMC Pharmacol Toxicol. 2018;19(1):81.

33. Pomorska A, et al. Isavuconazole in a successful combination treatment of disseminated mucormycosis in a child with acute lymphoblastic leukaemia and generalized haemochromatosis: a case report and review of the literature. Mycopathologia. 2019;184(1):81-8.

Publisher's Note Springer Nature remains neutral with regard to jurisdictional claims in published maps and institutional affiliations. 